\title{
Trivalent $f$-Element Squarates, Squarate-Oxalates, Cationic Materials, and the Determination of the Nine-Coordinate Ionic Radius of Cf(III)
}

Natasha Brenner, ${ }^{1}$ Joseph Sperling, ${ }^{2}$ Todd N. Poe, ${ }^{1,2}$ Cristian Celis-Barros ${ }^{2}$, Kristi Brittain, ${ }^{1}$ Eric M. Villa, ${ }^{3}$ Thomas E. Albrecht-Schmitt,$^{2}$ and Matthew J. Polinski ${ }^{*}$

${ }^{1}$ Department of Chemistry and Biochemistry, Bloomsburg University of Pennsylvania, 400 E. $2^{\text {nd }}$ Street Bloomsburg, PA. 17815, United States

${ }^{2}$ Department of Chemistry and Biochemistry, Florida State University, 95 Chieftan Way, Tallahassee, Florida 32306, United States

${ }^{3}$ Department of Chemistry, Creighton University, 2500 California Plaza, Omaha, Nebraska 68178, United States

E-mail: polinskimj@gmail.com

\section{Supporting Information}

Table of Contents

Selected bond distances for $\mathrm{M}_{2}\left(\mathrm{C}_{4} \mathrm{O}_{4}\right)_{3}\left(\mathrm{H}_{2} \mathrm{O}\right)_{2}(\mathrm{M}=\mathrm{Eu}, \mathrm{Am}, \mathrm{Cf})$

S2

Selected bond distances for $\mathrm{M}_{2}\left(\mathrm{C}_{4} \mathrm{O}_{4}\right)_{2}\left(\mathrm{C}_{2} \mathrm{O}_{4}\right)\left(\mathrm{H}_{2} \mathrm{O}\right)_{4}(\mathrm{M}=\mathrm{Am}, \mathrm{Cf})$

S3

Selected bond distances for $\mathrm{Sm}\left(\mathrm{C}_{4} \mathrm{O}_{4}\right)\left(\mathrm{C}_{4} \mathrm{O}_{3} \mathrm{OH}\right)\left(\mathrm{H}_{2} \mathrm{O}\right)_{2} \cdot 0.5 \mathrm{H}_{2} \mathrm{O}$ and

$\left[\mathrm{M}_{4}\left(\mathrm{C}_{4} \mathrm{O}_{4}\right)_{5}\left(\mathrm{H}_{2} \mathrm{O}\right)_{12}\right] \mathrm{Cl}_{2} \cdot 5 \mathrm{H}_{2} \mathrm{O}(\mathrm{M}=\mathrm{Eu}, \mathrm{Dy}, \mathrm{Ho}, \mathrm{Er})$

Bond Valence Sum Data for $\mathrm{Sm}\left(\mathrm{C}_{4} \mathrm{O}_{4}\right)\left(\mathrm{C}_{4} \mathrm{O}_{3} \mathrm{OH}\right)\left(\mathrm{H}_{2} \mathrm{O}\right)_{2} \cdot 0.5 \mathrm{H}_{2} \mathrm{O}$

S5

Data used for the linear regression fit in determining the nine-coordinate ionic radius of $\mathbf{C f}^{3+}$

References 
Table S1. Selected bond distances $(\AA)$ for $\mathrm{M}_{2}\left(\mathrm{C}_{4} \mathrm{O}_{4}\right)_{3}\left(\mathrm{H}_{2} \mathrm{O}\right)_{2}(\mathrm{M}=\mathrm{Eu}, \mathrm{Am}, \mathrm{Cf}$; Eu1, Am1, Cf1).

\begin{tabular}{|c|c|c|c|c|}
\hline Distance $(\AA)$ & \multicolumn{2}{|c|}{ Distance $(\AA)$} & \multicolumn{2}{|l|}{ Distance $(\AA)$} \\
\hline $\operatorname{Am}(1)-\mathrm{O}(1 \mathrm{w}) 2.514(9)$ & $\mathrm{Cf}(1)-\mathrm{O}(1 \mathrm{w})$ & $2.504(8)$ & $\mathrm{Eu}(1)-\mathrm{O}(1 \mathrm{w})$ & $2.433(6)$ \\
\hline $\mathrm{Am}(1)-\mathrm{O}(1) \quad 2.558(9)$ & $\mathrm{Cf}(1)-\mathrm{O}(1)$ & $2.515(7)$ & $\mathrm{Eu}(1)-\mathrm{O}(1)$ & $2.334(4)$ \\
\hline $\mathrm{Am}(1)-\mathrm{O}(2)$ & $\mathrm{Cf}(1)-\mathrm{O}(2)$ & $2.395(6)$ & $\mathrm{Eu}(1)-\mathrm{O}(2)$ & $2.411(4)$ \\
\hline $\mathrm{Am}(1)-\mathrm{O}(2) \quad 2.664(8)$ & $\mathrm{Cf}(1)-\mathrm{O}(2)$ & $2.656(6)$ & $\mathrm{Eu}(1)-\mathrm{O}(2)$ & $2.670(4)$ \\
\hline $\mathrm{Am}(1)-\mathrm{O}(2 \mathrm{w}) 2.469(8)$ & $\mathrm{Cf}(1)-\mathrm{O}(2 \mathrm{w})$ & $2.436(7)$ & $\mathrm{Eu}(1)-\mathrm{O}(2 \mathrm{w})$ & $2.486(4)$ \\
\hline $\mathrm{Am}(1)-\mathrm{O}(3) \quad 2.426(9)$ & $\mathrm{Cf}(1)-\mathrm{O}(3)$ & $2.378(7)$ & $\mathrm{Eu}(1)-\mathrm{O}(3)$ & $2.456(4)$ \\
\hline $\mathrm{Am}(1)-\mathrm{O}(5)$ & $\mathrm{Cf}(1)-\mathrm{O}(5)$ & $2.466(7)$ & $\mathrm{Eu}(1)-\mathrm{O}(5)$ & $2.375(4)$ \\
\hline $\mathrm{Am}(1)-\mathrm{O}(5)$ & $\mathrm{Cf}(1)-\mathrm{O}(5)$ & $2.767(7)$ & $\mathrm{Eu}(1)-\mathrm{O}(5)$ & $2.837(4)$ \\
\hline $\operatorname{Am}(1)-\mathrm{O}(6)$ & $\mathrm{Cf}(1)-\mathrm{O}(6)$ & $2.315(7)$ & $\mathrm{Eu}(1)-\mathrm{O}(6)$ & $2.509(5)$ \\
\hline
\end{tabular}


Table S2. Selected bond distances ( $\AA$ ) for $\mathrm{Am}_{2}\left(\mathrm{C}_{4} \mathrm{O}_{4}\right)_{2}\left(\mathrm{C}_{2} \mathrm{O}_{4}\right)\left(\mathrm{H}_{2} \mathrm{O}\right)_{4}$ (Am2) and $\mathrm{Cf}_{2}\left(\mathrm{C}_{4} \mathrm{O}_{4}\right)_{2}\left(\mathrm{C}_{2} \mathrm{O}_{4}\right)\left(\mathrm{H}_{2} \mathrm{O}\right)_{4}(\mathbf{C f} 2)$.

\begin{tabular}{|c|c|c|}
\hline Distance $(\AA)$ & Distar & ce $(\AA)$ \\
\hline $\operatorname{Am}(1)-\mathrm{O}(1 \mathrm{w}) 2.469(3)$ & $\mathrm{Cf}(1)-\mathrm{O}(1 \mathrm{w})$ & $2.455(4)$ \\
\hline $\mathrm{Am}(1)-\mathrm{O}(1) \quad 2.473(3)$ & $\mathrm{Cf}(1)-\mathrm{O}(1)$ & $2.400(4)$ \\
\hline $\mathrm{Am}(1)-\mathrm{O}(2 \mathrm{w}) 2.477(3)$ & $\mathrm{Cf}(1)-\mathrm{O}(2 \mathrm{w})$ & $2.452(5)$ \\
\hline $\mathrm{Am}(1)-\mathrm{O}(2) \quad 2.465(3)$ & $\mathrm{Cf}(1)-\mathrm{O}(2)$ & $2.446(5)$ \\
\hline $\mathrm{Am}(1)-\mathrm{O}(3)$ & $\mathrm{Cf}(1)-\mathrm{O}(3)$ & $2.463(4)$ \\
\hline $\mathrm{Am}(1)-\mathrm{O}(4)$ & $\mathrm{Cf}(1)-\mathrm{O}(4)$ & $2.424(5)$ \\
\hline $\mathrm{Am}(1)-\mathrm{O}(5)$ & $\mathrm{Cf}(1)-\mathrm{O}(5)$ & $2.434(4)$ \\
\hline $\mathrm{Am}(1)-\mathrm{O}(6)$ & $\mathrm{Cf}(1)-\mathrm{O}(6)$ & $2.372(4)$ \\
\hline $\mathrm{Am}(1)-\mathrm{O}(6)$ & $\mathrm{Cf}(1)-\mathrm{O}(6)$ & $2.851(5)$ \\
\hline
\end{tabular}


Table S3. Selected bond distances $(\AA)$ for $\mathrm{Sm}\left(\mathrm{C}_{4} \mathrm{O}_{4}\right)\left(\mathrm{C}_{4} \mathrm{O}_{3} \mathrm{OH}\right)\left(\mathrm{H}_{2} \mathrm{O}\right)_{2} \cdot 0.5 \mathrm{H}_{2} \mathrm{O}$ ( $\mathbf{S m 3}$ ), $\left[\mathrm{Eu}_{4}\left(\mathrm{C}_{4} \mathrm{O}_{4}\right)_{5}\left(\mathrm{H}_{2} \mathrm{O}\right)_{12}\right] \mathrm{Cl}_{2} \cdot 5 \mathrm{H}_{2} \mathrm{O} \quad(\mathbf{E u} 4), \quad\left[\mathrm{Dy}_{4}\left(\mathrm{C}_{4} \mathrm{O}_{4}\right)_{5}\left(\mathrm{H}_{2} \mathrm{O}\right)_{12}\right] \mathrm{Cl}_{2} \cdot 5 \mathrm{H}_{2} \mathrm{O} \quad($ Dy4 $)$, $\left[\mathrm{Ho}_{4}\left(\mathrm{C}_{4} \mathrm{O}_{4}\right)_{5}\left(\mathrm{H}_{2} \mathrm{O}\right)_{12}\right] \mathrm{Cl}_{2} \cdot 5 \mathrm{H}_{2} \mathrm{O}(\mathbf{H o 4})$, and $\left[\mathrm{Er}_{4}\left(\mathrm{C}_{4} \mathrm{O}_{4}\right)_{5}\left(\mathrm{H}_{2} \mathrm{O}\right)_{12}\right] \mathrm{Cl}_{2} \cdot 5 \mathrm{H}_{2} \mathrm{O}(\mathbf{E r 4})$.

\begin{tabular}{|c|c|c|c|c|c|}
\hline \multicolumn{4}{|c|}{ Distance $(\AA)$} & \multirow[b]{2}{*}{$\mathrm{Eu}(2)-\mathrm{O}(2)$} & \multirow[b]{2}{*}{$2.517(5)$} \\
\hline $\mathrm{Sm}(1)-\mathrm{O}(1 \mathrm{w})$ & $2.500(2)$ & $\mathrm{Eu}(1)-\mathrm{O}(1)$ & $2.330(5)$ & & \\
\hline $\mathrm{Sm}(1)-\mathrm{O}(1)$ & $2.413(2)$ & $\mathrm{Eu}(1)-\mathrm{O}(3)$ & $2.348(5)$ & $\mathrm{Eu}(2)-\mathrm{O}(4)$ & $2.413(5)$ \\
\hline $\mathrm{Sm}(1)-\mathrm{O}(2 \mathrm{w})$ & $2.461(2)$ & $\mathrm{Eu}(1)-\mathrm{O}(5)$ & $2.428(5)$ & $\mathrm{Eu}(2)-\mathrm{O}(6)$ & $2.434(5)$ \\
\hline $\mathrm{Sm}(1)-\mathrm{O}(3)$ & $2.426(2)$ & $\mathrm{Eu}(1)-\mathrm{O}(8)$ & $2.435(5)$ & $\mathrm{Eu}(2)-\mathrm{O}(7)$ & $2.381(5)$ \\
\hline $\mathrm{Sm}(1)-\mathrm{O}(5)$ & $2.364(2)$ & $\mathrm{Eu}(1)-\mathrm{O}(9)$ & $2.444(4)$ & $\mathrm{Eu}(2)-\mathrm{O}(12)$ & $2.392(5)$ \\
\hline $\mathrm{Sm}(1)-\mathrm{O}(6)$ & $2.440(2)$ & $\mathrm{Eu}(1)-\mathrm{O}(10)$ & $2.364(5)$ & $\mathrm{Eu}(2)-\mathrm{O}(13)$ & $2.401(5)$ \\
\hline $\mathrm{Sm}(1)-\mathrm{O}(7)$ & $2.389(2)$ & $\mathrm{Eu}(1)-\mathrm{O}(11)$ & $2.448(5)$ & $\mathrm{Eu}(2)-\mathrm{O}(14)$ & $2.375(4)$ \\
\hline $\mathrm{Sm}(1)-\mathrm{O}(8)$ & $2.408(2)$ & $\mathrm{Eu}(1)-\mathrm{O}(16)$ & $2.437(5)$ & $\mathrm{Eu}(2)-\mathrm{O}(15)$ & $2.385(5)$ \\
\hline $\mathrm{Dy}(1)-\mathrm{O}(1)$ & $2.298(9)$ & $\mathrm{Dy}(2)-\mathrm{O}(2)$ & $2.426(12)$ & & \\
\hline Dy(1)-O(3) & $2.298(10)$ & $\mathrm{Dy}(2)-\mathrm{O}(4)$ & $2.422(12)$ & & \\
\hline Dy(1)-O(5) & $2.398(9)$ & $\operatorname{Dy}(2)-O(6)$ & $2.353(13)$ & & \\
\hline Dy(1)-O(8) & $2.372(10)$ & Dy(2)-O(7) & $2.335(10)$ & & \\
\hline Dy(1)-O(9) & $2.433(11)$ & $\mathrm{Dy}(2)-\mathrm{O}(12)$ & $2.347(11)$ & & \\
\hline Dy(1)-O(10) & $2.307(9)$ & $\mathrm{Dy}(2)-\mathrm{O}(13)$ & $2.327(10)$ & & \\
\hline Dy(1)-O(11) & $2.409(10)$ & Dy(2)-O(14) & $2.316(8)$ & & \\
\hline Dy(1)-O(16) & $2.410(9)$ & Dy $(2)-O(15)$ & $2.343(9)$ & & \\
\hline $\mathrm{Ho}(1)-\mathrm{O}(1)$ & $2.303(9)$ & $\mathrm{Ho}(2)-\mathrm{O}(2)$ & $2.410(11)$ & & \\
\hline $\mathrm{Ho}(1)-\mathrm{O}(3)$ & $2.262(8)$ & $\mathrm{Ho}(2)-\mathrm{O}(4)$ & $2.370(12)$ & & \\
\hline $\mathrm{Ho}(1)-\mathrm{O}(5)$ & $2.377(9)$ & $\mathrm{Ho}(2)-\mathrm{O}(6)$ & $2.333(11)$ & & \\
\hline $\mathrm{Ho}(1)-\mathrm{O}(8)$ & $2.344(9)$ & $\mathrm{Ho}(2)-\mathrm{O}(7)$ & $2.352(10)$ & & \\
\hline $\mathrm{Ho}(1)-\mathrm{O}(9)$ & $2.398(11)$ & $\mathrm{Ho}(2)-\mathrm{O}(12)$ & $2.330(9)$ & & \\
\hline $\mathrm{Ho}(1)-\mathrm{O}(10)$ & $2.289(9)$ & $\mathrm{Ho}(2)-\mathrm{O}(13)$ & $2.337(10)$ & & \\
\hline $\mathrm{Ho}(1)-\mathrm{O}(11)$ & 2.391(11) & $\mathrm{Ho}(2)-\mathrm{O}(14)$ & $2.317(9)$ & & \\
\hline $\mathrm{Ho}(1)-\mathrm{O}(16)$ & $2.373(9)$ & $\mathrm{Ho}(2)-\mathrm{O}(15)$ & $2.317(9)$ & & \\
\hline $\operatorname{Er}(1)-\mathrm{O}(1)$ & $2.270(8)$ & $\mathrm{Er}(2)-\mathrm{O}(2)$ & $2.394(12)$ & & \\
\hline $\mathrm{Er}(1)-\mathrm{O}(3)$ & $2.266(9)$ & $\mathrm{Er}(2)-\mathrm{O}(4)$ & $2.383(12)$ & & \\
\hline $\mathrm{Er}(1)-\mathrm{O}(5)$ & $2.359(8)$ & $\operatorname{Er}(2)-\mathrm{O}(6)$ & $2.308(12)$ & & \\
\hline $\operatorname{Er}(1)-\mathrm{O}(8)$ & $2.348(10)$ & $\operatorname{Er}(2)-\mathrm{O}(7)$ & $2.340(10)$ & & \\
\hline $\mathrm{Er}(1)-\mathrm{O}(9)$ & $2.400(11)$ & $\operatorname{Er}(2)-O(12)$ & $2.324(10)$ & & \\
\hline $\operatorname{Er}(1)-\mathrm{O}(10)$ & $2.292(9)$ & $\operatorname{Er}(2)-O(13)$ & $2.325(11)$ & & \\
\hline $\operatorname{Er}(1)-\mathrm{O}(11)$ & $2.399(11)$ & $\operatorname{Er}(2)-O(14)$ & $2.299(8)$ & & \\
\hline $\operatorname{Er}(1)-\mathrm{O}(16)$ & $2.368(9)$ & $\operatorname{Er}(2)-\mathrm{O}(15)$ & $2.322(9)$ & & \\
\hline
\end{tabular}


Figure S1. Bond Valence Sum (BVS) data for $\mathrm{Sm}\left(\mathrm{C}_{4} \mathrm{O}_{4}\right)\left(\mathrm{C}_{4} \mathrm{O}_{3} \mathrm{OH}\right)\left(\mathrm{H}_{2} \mathrm{O}\right)_{2} \cdot 0.5 \mathrm{H}_{2} \mathrm{O}(\mathbf{S m 3})$. BVS parameters provide complimentary support for the assignment of water and hydroxyl groups in the structure. Specific parameters and values for each atom were obtained from Refs. $1-3$.

\begin{tabular}{|c|c|c|c|c|c|c|c|c|c|c|c|}
\hline & $v u=\exp [(R 0-d) / b]$ & & & & & & & & & & \\
\hline Squarate & & & distance & & & & & distance & & Total & \\
\hline$R(0): C-O$ & 1.39 & $\mathrm{C}(1)-\mathrm{O}(1)$ & 1.253 & 1.448 & $R(0): S m-O$ & 2.063 & $\mathrm{Sm}(1)-\mathrm{O}(1)$ & 2.413 & 0.388 & 1.836 & \\
\hline b & 0.37 & $\mathrm{C}(2)-\mathrm{O}(2)$ & 1.317 & 1.218 & b & 0.37 & & & & 1.218 & $\mathrm{OH}$ \\
\hline & & $\mathrm{C}(3)-\mathrm{O}(3)$ & 1.244 & 1.484 & & & $\mathrm{Sm}(1)-\mathrm{O}(3)$ & 2.426 & 0.375 & 1.859 & \\
\hline & & $\mathrm{C}(4)-\mathrm{O}(4)$ & 1.227 & 1.554 & & & & & & 1.554 & \\
\hline & & $\mathrm{C}(5)-\mathrm{O}(5)$ & 1.242 & 1.492 & & & $\mathrm{Sm}(1)-\mathrm{O}(5)$ & 2.364 & 0.443 & 1.935 & \\
\hline & & $\mathrm{C}(6)-\mathrm{O}(6)$ & 1.264 & 1.406 & & & $\mathrm{Sm}(1)-\mathrm{O}(6)$ & 2.44 & 0.361 & 1.406 & \\
\hline & & $\mathrm{C}(7)-\mathrm{O}(7)$ & 1.243 & 1.488 & & & $\mathrm{Sm}(1)-\mathrm{O}(7)$ & 2.389 & 0.414 & 1.902 & \\
\hline & & $\mathrm{C}(8)-\mathrm{O}(8)$ & 1.26 & 1.421 & & & $\mathrm{Sm}(1)-\mathrm{O}(8)$ & 2.408 & 0.394 & 1.815 & \\
\hline & & & & & & & $\operatorname{Sm}(1)-O(1 w)$ & 2.5 & 0.307 & 0.307 & $\mathrm{H} 2 \mathrm{O}$ \\
\hline & & & & & & & $\operatorname{Sm}(1)-O(2 w)$ & 2.461 & 0.341 & 0.341 & $\mathrm{H} 2 \mathrm{O}$ \\
\hline & & & & & & & & & & & \\
\hline & & & & & & & & & & & \\
\hline & & & & & $\mathrm{R}(0): \mathrm{Sm}-\mathrm{O}$ & 2.063 & $\mathrm{Sm}(1)-\mathrm{O}(1)$ & 2.413 & 0.388 & & \\
\hline & & & & & b & 0.37 & $\mathrm{Sm}(1)-\mathrm{O}(3)$ & 2.426 & 0.375 & & \\
\hline & & & & & & & $\mathrm{Sm}(1)-\mathrm{O}(5)$ & 2.364 & 0.443 & & \\
\hline & & & & & & & $\mathrm{Sm}(1)-\mathrm{O}(6)$ & 2.44 & 0.361 & & \\
\hline & & & & & & & $\mathrm{Sm}(1)-\mathrm{O}(7)$ & 2.389 & 0.414 & & \\
\hline & & & & & & & $\mathrm{Sm}(1)-\mathrm{O}(8)$ & 2.408 & 0.394 & & \\
\hline & & & & & & & $\operatorname{Sm}(1)-O(1 w)$ & 2.5 & 0.307 & & \\
\hline & & & & & & & $\operatorname{Sm}(1)-O(2 w)$ & 2.461 & 0.341 & & \\
\hline & & & & & & & & & & & \\
\hline & & & & & & & & & & & \\
\hline & & & & & & & Sm(1) BVS & & 3.023 & & \\
\hline
\end{tabular}


Figure S2. Data used for the linear regression fit in determining the nine-coordinate ionic radius of $\mathrm{Cf}^{3+}$. The nine-coordinate ionic radii and unit cell volumes as a function of the lanthanides comprising the isotypic series $\mathrm{M}_{2}\left(\mathrm{C}_{4} \mathrm{O}_{4}\right)_{3}\left(\mathrm{H}_{2} \mathrm{O}\right)_{4}(\mathrm{M}=\mathrm{La}-\mathrm{Eu})$. All nine-coordinate ionic radii obtained from Ref. 4.

\begin{tabular}{|c|c|c|}
\hline $\mathbf{R E}^{3+}$ & Unit Cell V $\left.\mathbf{( \AA}^{\mathbf{3}}\right)$ & Ionic Radius $(\AA \mathbf{\AA})$ \\
\hline $\mathrm{La}$ & 838.03 & 1.216 \\
\hline $\mathrm{Ce}$ & 826.79 & 1.196 \\
\hline $\mathrm{Pr}$ & 819.97 & 1.179 \\
\hline $\mathrm{Nd}$ & 811.65 & 1.163 \\
\hline $\mathrm{Sm}$ & 800.78 & 1.132 \\
\hline $\mathrm{Eu}$ & 796.53 & 1.120 \\
\hline $\mathrm{Cf}$ & 798.5 & $? ? ?$ \\
\hline
\end{tabular}




\section{References}

1. Brown, I. D. Recent Developments in the Methods and Applications of the Bond Valence Model. Chem. Rev. 2009, 109, 6858-6919.

2. Brown, I. D.; Altermatt, D. Bond-Valence Parameters Obtained from a Systematic Analysis of the Inorganic Crystal Structure Database. Acta Cryst. 1985, B41, 244-247.

3. Brese, N. E.; O’Keefe, M. Bond-Valence Parameters for Solids. Acta Cryst. 1991, B47, 192-97.

4. Shannon, R. D. Revised Effective Ionic Radii and Systematic Studies of Interatomic Distances in Halides and Chalcogenides. Acta Crystallogr., Sect. A. 1976, 32, 751-767. 